\title{
The QGP Equation of State by Measuring the Color Suppression Factor at RHIC and LHC Energies
}

\section{R. P. Scharenberg*}

Department of Physics, Purdue University, West Lafayette, Indiana-47907, USA

E-mail: schrnbrg@purdue.edu

\begin{abstract}
The initial temperature $T_{i}$, energy density $\varepsilon_{i}$, and formation time $\tau_{i}$ of the initial state of the QGP formed in the heavy ion collisions at RHIC and LHC energies are determined using the data driven Color String Percolation Model (CSPM). Multiparticle production by interacting strings stretched between projectile and target form a spanning cluster at the percolation threshold. The relativistic kinetic theory relation for $\eta / s$ is evaluated as a function of $T$ and the mean free path $\left(\lambda_{m f p}\right)$ using data and CSPM. $\eta / s\left(T_{i}, \lambda_{m f p}\right)$ describes the transition from a strongly interacting QGP at $T / T_{c} \sim 1$ to a weakly coupled QGP at $T / T_{c} \geq 6$. We find that the reciprocal of $\eta / s$ is equal to the trace anomaly $\Delta=\varepsilon-3 P / T^{4}$ which also describes the transition. We couple this initial state of the QGP to a 1D Bjorken expansion to determine the sound velocity $c_{s}^{2}$ of the QGP for $1 \leq T / T_{c} \leq 3$. The bulk thermodynamic quantities and the equation of state are in excellent agreement with LQCD results.
\end{abstract}

8th International Workshop on Critical Point and Onset of Deconfinement,

March 11 to 15, 2013

Napa, California, USA

*Speaker. 


\section{Clustering of Color Sources}

Multiparticle production is currently described in terms of color strings stretched between the projectile and the target, which decay into new strings and subsequently hadronize to produce observed hadrons. Color strings may be viewed as small areas in the transverse plane filled with color field created by colliding partons. With growing energy and size of the colliding system, the number of strings grows, and they start to overlap, forming clusters, in the transverse plane very much similar to disks in two dimensional percolation theory. At a certain critical density a macroscopic cluster appears that marks the percolation phase transition. This is the Color String Percolation Model (CSPM) [1, 2]. The interaction between strings occurs when they overlap and the general result, due to the $\mathrm{SU}(3)$ random summation of charges, is a reduction in multiplicity and an increase in the string tension hence increase in the average transverse momentum squared, $\left\langle p_{t}^{2}\right\rangle$.

CSPM can connect the multiplicity $\mu$ and the mean transverse momentum squared $\left\langle p_{t}^{2}\right\rangle$ of the particles produced by a cluster of $n$ strings [2]

$$
\mu_{n}=\sqrt{\frac{n S_{n}}{S_{1}}} \mu_{0} ; \quad\left\langle p_{t}^{2}\right\rangle=\sqrt{\frac{n S_{1}}{S_{n}}}\left\langle p_{t}^{2}\right\rangle_{1}
$$

where $\mu_{0}$ and $\left\langle p_{t}^{2}\right\rangle_{1}$ are the mean multiplicity and $\left\langle p_{t}^{2}\right\rangle$ of particles produced from a single string with a transverse area $S_{1}=\pi r_{0}^{2}$. This implies a simple relation between the multiplicity and transverse momentum $\mu_{n}\left\langle p_{t}^{2}\right\rangle_{n}=n \mu_{0}\left\langle p_{t}^{2}\right\rangle_{1}$, which means conservation of the total transverse momentum produced.

In the thermodynamic limit, one obtains an analytic expression $[1,2]$

$$
\left\langle\frac{n S_{1}}{S_{n}}\right\rangle=\frac{\xi}{1-e^{-\xi}} \equiv \frac{1}{F(\xi)^{2}}
$$

where $F(\xi)$ is the color suppression factor and $\xi=\frac{N_{s} S_{1}}{S_{N}}$ is the percolation density parameter. Eq.(1.1) can be written as $\mu_{n}=n F(\xi) \mu_{0}$ and $\left\langle p_{t}^{2}\right\rangle_{n}=\left\langle p_{t}^{2}\right\rangle_{1} / F(\xi)$. The critical cluster which spans $S_{N}$, appears for $\xi_{c} \geq 1.2$ [3]. It is worth noting that CSPM is a saturation model similar to the Color Glass Condensate (CGC), where $\left\langle p_{t}^{2}\right\rangle_{1} / F(\xi)$ plays the same role as the saturation momentum scale $Q_{s}^{2}$ in the CGC model $[4,5]$.

\section{Experimental Determination of the Color Suppression Factor $F(\xi)$}

The suppression factor is determined by comparing the $p p$ and $\mathrm{A}+\mathrm{A}$ transverse momentum spectra. To evaluate the value of $\xi$ from data for $\mathrm{Au}+\mathrm{Au}$ collisions, a parameterization of $p p$ events at $200 \mathrm{GeV}$ is used to compute the $p_{t}$ distribution

$$
d N_{c} / d p_{t}^{2}=a /\left(p_{0}+p_{t}\right)^{\alpha}
$$

where a is the normalization factor. $p_{0}$ and $\alpha$ are parameters used to fit the data. This parameterization also can be used for nucleus-nucleus collisions to take into account the interactions of the strings [2]

$$
d N_{c} / d p_{t}^{2}=\frac{a^{\prime}}{\left(p_{0} \sqrt{F\left(\xi_{p p}\right) / F(\xi)}+p_{t}\right)^{\alpha}}
$$


The color suppression factor $F(\xi)$ is related to the percolation density parameter $\xi$.

$$
F(\xi)=\sqrt{\frac{1-e^{-\xi}}{\xi}}
$$

\section{Determination of the Temperature}

The connection between the measured $\xi$ and the temperature $T(\xi)$ involves the Schwinger mechanism (SM) for particle production. The Schwinger distribution for massless particles is expressed in terms of $p_{t}^{2}[6,7]$

$$
d n / d p_{t}^{2} \sim e^{-\pi p_{t}^{2} / x^{2}}
$$

where the average value of the string tension is $\left\langle x^{2}\right\rangle$. The tension of the macroscopic cluster fluctuates around its mean value because the chromo-electric field is not constant. The origin of the string fluctuation is related to the stochastic picture of the QCD vacuum. Since the average value of the color field strength must vanish, it can not be constant but changes randomly from point to point [8]. Such fluctuations lead to a Gaussian distribution of the string tension for the cluster, which transforms SM into the thermal distribution.

$$
d n / d p_{t}^{2} \sim e^{\left(-p_{t} \sqrt{\frac{2 \pi}{\left\langle x^{2}\right\rangle}}\right)}
$$

with $\left\langle x^{2}\right\rangle=\pi\left\langle p_{t}^{2}\right\rangle_{1} / F(\xi)$. The temperature is expressed as $[9,10]$

$$
T(\xi)=\sqrt{\frac{\left\langle p_{t}^{2}\right\rangle_{1}}{2 F(\xi)}}
$$

We adopt the point of view that the experimentally determined universal chemical freeze-out temperature $\left(T_{f}\right)$ is a good measure of the phase transition temperature, $T_{c}$. $\left\langle p_{t}^{2}\right\rangle_{1}$ is evaluated using Eq.(3.3) at $\xi_{c}=1.2$ with $T_{f}=167.7 \pm 2.6 \mathrm{MeV}$ [11]. This gives $\sqrt{\left\langle p_{t}^{2}\right\rangle_{1}}=207.2 \pm 3.3 \mathrm{MeV}$. This calibrates the CSPM temperature scale.

\section{Energy Density $\varepsilon$}

The initial energy density $\varepsilon_{i}$ above $T_{c}$ is given by [12]

$$
\varepsilon_{i}=\frac{3}{2} \frac{\frac{d N_{c}}{d y}\left\langle m_{t}\right\rangle}{S_{n} \tau_{\text {pro }}}
$$

To evaluate $\varepsilon_{i}$ we use the charged pion multiplicity $d N_{c} / d y$ at midrapidity and $S_{n}$ values from STAR for $0-10 \%$ central Au-Au collisions with $\sqrt{s_{N N}}=200 \mathrm{GeV}$ [13]. We can calculate $\left\langle p_{t}\right\rangle$ using the CSPM thermal distribution Eqs. (3.2) and (3.3). For $0.2<p_{t}<1.5,\left\langle p_{t}\right\rangle=0.394 \pm$ $0.003 \mathrm{GeV}$, adding the extra energy required for the rest mass of pions at hadronization $\left\langle m_{t}\right\rangle=$ $0.42 \pm 0.003 \mathrm{GeV}$. The error on $\left\langle p_{t}\right\rangle$ is due to the error on $T_{i}[10]$.

The dynamics of massless particle production has been studied in QE2 quantum electrodynamics. QE2 can be scaled from electrodynamics to quantum chromodynamics using the ratio of the coupling constants [6]. The production time $\tau_{\text {pro }}$ for a boson (gluon) is [7]

$$
\tau_{\text {pro }}=\frac{2.405 \hbar}{\left\langle m_{t}\right\rangle}
$$


For Au-Au collisions at $\sqrt{s_{N N}}=200 \mathrm{GeV}$ Eqs.(4.1) and (4.2) gives $\varepsilon_{i}=2.27 \pm 0.16 \mathrm{GeV} / \mathrm{fm}^{3}$ at $\xi=2.88$. In CSPM the energy density $\varepsilon$ is proportional to $\xi$. From the measured value of $\xi$ and $\varepsilon$ it is found that $\varepsilon$ is proportional to $\xi$ for the range $1.2<\xi<10.56, \varepsilon_{i}=0.788 \xi \mathrm{GeV} / \mathrm{fm}^{3}[13,14]$ as shown in Fig.1.

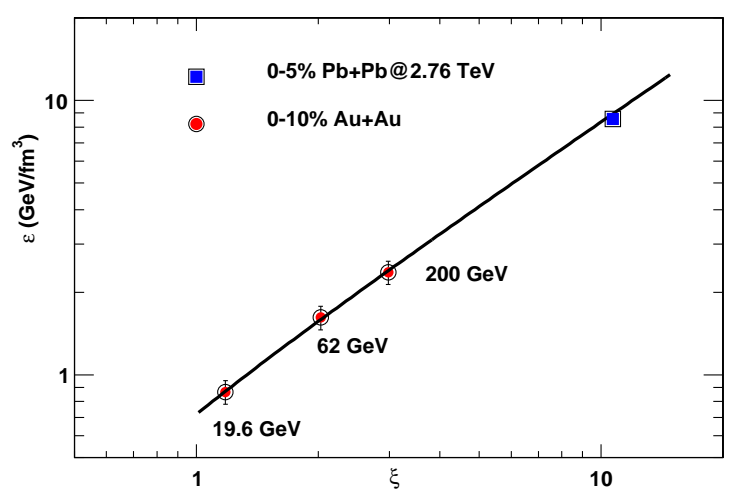

Figure 1: Energy density $\varepsilon$ as a function of the percolation density parameter $\xi$. The value for LHC energy is shown as blue square $[13,14]$.

Figure 2 shows a plot of $\varepsilon / T^{4}$ as a function of $\mathrm{T} / T_{c}$. The lattice QCD results are from HotQCD Collaboration [15]. It is observed that at LHC energy the CSPM results are in excellent agreement with the lattice QCD results. The lattice and CSPM results are available for $\mathrm{T} / T_{c}<2$.

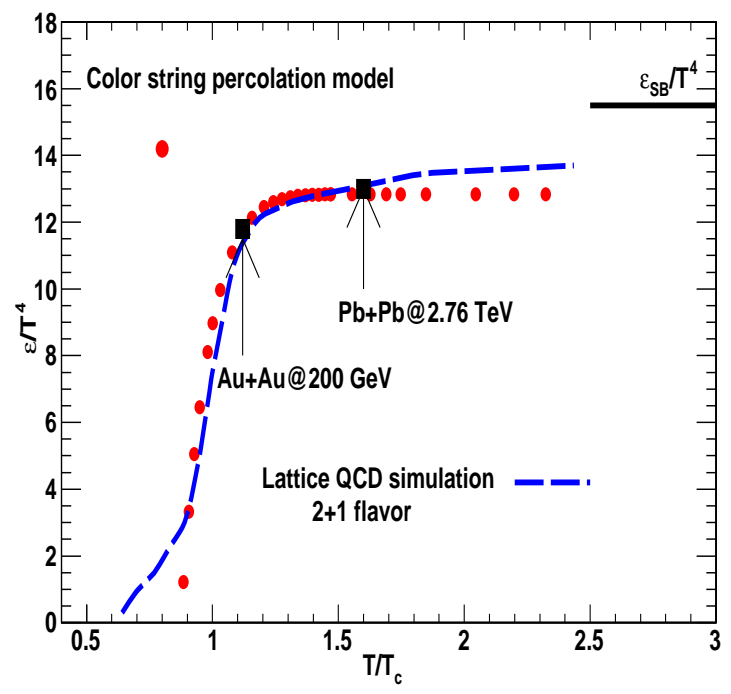

Figure 2: The energy density from CSPM versus $T / T_{c}^{C S P M}$ (red circles) and Lattice QCD energy density vs $T / T_{c}^{L Q C D}$ (blue dash line) for $2+1$ flavor and $\mathrm{p} 4$ action [15].

\section{The Shear Viscosity to Entropy Density ratio $\eta / s$}

The relativistic kinetic theory relation for the shear viscosity over entropy density ratio, $\eta / s$ is 
given by $[16,17]$

$$
\frac{\eta}{s} \simeq \frac{T \lambda_{m f p}}{5}
$$

where $\mathrm{T}$ is the temperature and $\lambda_{m f p}$ is the mean free path given by

$$
\lambda_{m f p} \sim \frac{1}{\left(n \sigma_{t r}\right)}
$$

$n$ is the number density of an ideal gas of quarks and gluons and $\sigma_{t r}$ the transport cross section for these constituents.

After the cluster is formed it behaves like a free gas of constituents. Eq.(5.1) can be applied to obtain the shear viscosity. In CSPM the number density is given by the effective number of sources per unit volume [18]

$$
n=\frac{N_{\text {sources }}}{S_{N} L}
$$

$\mathrm{L}$ is the longitudinal extension of the source, $\mathrm{L}=1 \mathrm{fm}$ [9]. The area occupied by the strings is related to $\xi$ through the relation $\left(1-e^{-\xi}\right) S_{N}$. Thus the effective no. of sources is given by the total area occupied by the strings divided by the effective area of the string $S_{1} F(\xi)$.

$$
N_{\text {sources }}=\frac{\left(1-e^{-\xi}\right) S_{N}}{S_{1} F(\xi)}
$$

In general $N_{\text {sources }}$ is smaller than the number of single strings. The number density of sources from Eqs.(5.3) and (5.4) becomes

$$
n=\frac{\left(1-e^{-\xi}\right)}{S_{1} F(\xi) L}
$$

In CSPM the transport cross section $\sigma_{t r}$ is the transverse area of the effective string $S_{1} F(\xi)$. Thus $\sigma_{t r}$ is directly proportional to $F(\xi)$ and hence to $\frac{1}{T^{2}}$. The mean free path is given by

$$
\begin{gathered}
\lambda_{m f p}=\frac{L}{\left(1-e^{-\xi}\right)} \\
\frac{\eta}{s}=\frac{T L}{5\left(1-e^{-\xi}\right)}
\end{gathered}
$$

The behavior of $\eta / s$ is dominated by the fractional area covered by strings for $\xi<\xi_{c}$. This is not surprising because $\eta / s$ is the ability to transport momenta at large distances and that has to do with the density of voids in the matter.

The theoretical estimates of $\eta / s$ has been obtained as a function of $\mathrm{T} / T_{c}$ for both the weakly (wQGP) and strongly (sQGP) coupled QCD plasma are shown in Fig. 3 [16]. It is seen that at the RHIC top energy $\eta / s$ is close to the sQGP.

The $\Delta$ and $\eta / s$ both describe the transition from a strongly coupled QGP to a weakly coupled QGP. We find that the reciprocal of $\eta / s$ is in quantitative agreement with $\varepsilon-3 P / T^{4}$ the trace anomaly over this wide temperature range. This result is shown in Fig.4. The minimum in $\eta / s$ $=0.20$ at $T / T_{c} 1.15$ determines the peak of the interaction measure $\sim 5$ in agreement with the recent HotQCD values [21]. 


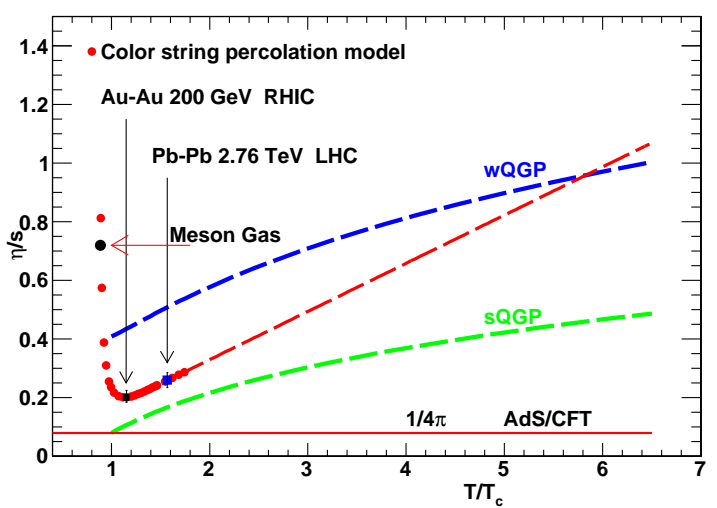

Figure 3: $\eta / s$ as a function of $\mathrm{T} / T_{c}$. Au+Au at $200 \mathrm{GeV}$ for $0-10 \%$ centrality is shown as solid black square. wQGP and sQGP values are shown as dotted blue and green lines respectively [16]. The red dotted line represents the extrapolation to higher temperatures from the CSPM. The hadron gas value for $\eta / s \sim 0.7$ is shown as solid black circle at $\mathrm{T} / T_{c} \sim 0.88$ [19]. The red solid line is Ads/CFT limit [20]

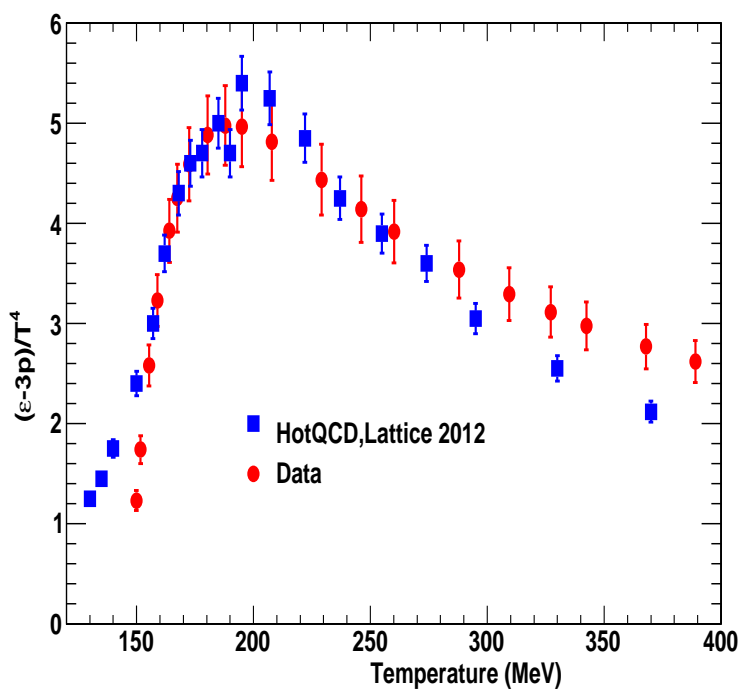

Figure 4: The trace anomaly $\Delta=\varepsilon-3 P / T^{4}$ vs temperature.

\section{The Equation of State}

We use CSPM coupled to a 1D Bjorken expansion. The input parameters are the initial temperature $\mathrm{T}$, the initial energy density $\varepsilon$, and the trace anomaly $\Delta$ are determined by data. The Bjorken $1 \mathrm{D}$ expansion gives the sound velocity

$$
\begin{aligned}
\frac{1}{T} \frac{d T}{d \tau} & =-C_{s}^{2} / \tau \\
\frac{d T}{d \tau} & =\frac{d T}{d \varepsilon} \frac{d \varepsilon}{d \tau} \\
\frac{d \varepsilon}{d \tau} & =-T s / \tau
\end{aligned}
$$

where $\varepsilon$ is the energy density, s the entropy density, $\tau$ the proper time, and $C_{s}$ the sound velocity. 
Since $s=\varepsilon+P / T$ and $P=\left(\varepsilon-\Delta T^{4}\right) / 3$ one gets

$$
\frac{d T}{d \varepsilon} s=C_{s}^{2}
$$

From above equations $C_{s}^{2}$ can be expressed in terms of $\xi$

$$
C_{s}^{2}=(-0.33)\left(\frac{\xi e^{-\xi}}{1-e^{-\xi}}-1\right)+0.0191(\Delta / 3)\left(\frac{\xi e^{-\xi}}{\left(1-e^{-\xi}\right)^{2}}-\frac{1}{1-e^{-\xi}}\right)
$$

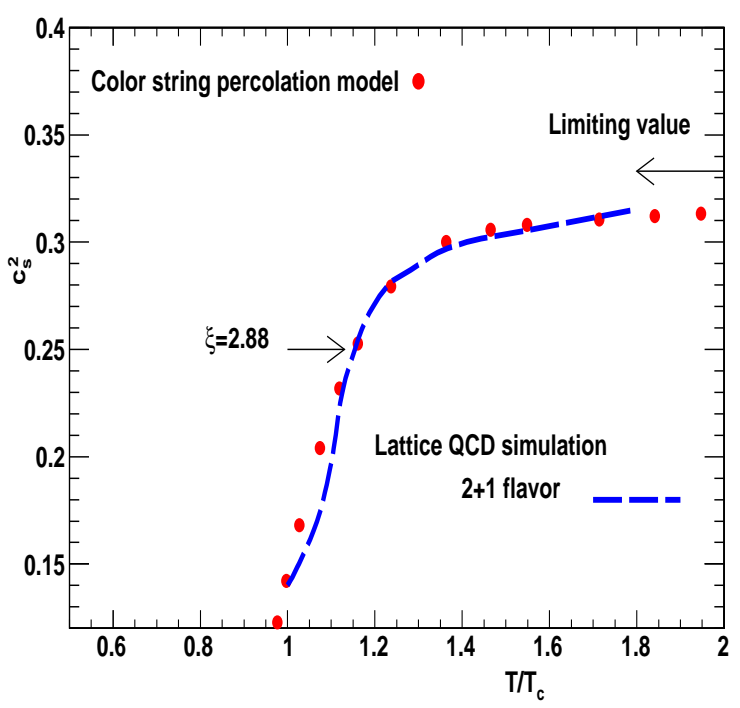

Figure 5: The speed of sound from CSPM versus $T / T_{C}^{C S P M}$ (red circles) and Lattice QCD-p4 speed of sound versus $T / T_{C}^{L Q C D}$ (blue dash line)[15].

\section{Conclusions and open questions}

Two central objectives in the experimental characterization of the QGP are the Equation Of State (EOS) and the shear viscosity to entropy ratio $\eta / s$. We have found that $s / \eta$ is equal to the trace anomaly $\Delta=\varepsilon-3 P / T^{4}$. We determine the bulk thermodynamics, $\varepsilon / T^{4}, s / T^{3}$ and the EOS $c_{s}^{2}$ of the QGP as a function of $T / T_{c}$ (CSPM). The results are in excellent agreement with LQCD numerical simulations as a function of $T / T_{C}(\mathrm{LQCD})$. CSPM predicts that the QGP will be formed in high multiplicity p-p collisions at LHC.

\section{Acknowledgments}

This research was supported by the Office of Nuclear Physics within the U.S. Department of Energy Office of Science under Grant No. DE-FG02-88ER40412. 


\section{References}

\section{References}

[1] M. A. Braun and C. Pajares 2000 Eur. Phys. J. C 16349.

[2] M. A. Braun, F. del Moral and C. Pajares 2002 Phys. Rev. C 65024907.

[3] H. Satz 2000 Rep. Prog. Phys. 631511.

[4] L. McLerran and R. Venugopalan 1994 Phys. Rev. D 492233.

[5] J. Dias de Deus and C. Pajares 2011 Phys. Lett. B 695455.

[6] C. Y. Wong, Introduction to high energy heavy ion collisions (World Scientific, 1994).

[7] J. Schwinger 1962 Phys. Rev. 1282425.

[8] A. Bialas 1999 Phys. Lett. B 466301.

[9] J. Dias de Deus and C. Pajares 2006 Phys. Lett. B 642455.

[10] R. P. Scharenberg, B. K. Srivastava and A. S. Hirsch 2011 Eur. Phys. J. C 711510.

[11] F. Becattini, P. Castorina, A. Milov and H. Satz 2010 Eur. Phys. J. C 66377.

[12] J. D. Bjorken 1983 Phys. Rev. D 27140.

[13] B. I. Abelev et al. (STAR Collaboration) 2009 Phys. Rev. C 7934909.

[14] B. K. Srivastava, R. P. Scharenberg, T. Tarnowsky, (STAR Collaboration) 2006 Nukleonika 51, s109.

[15] A. Bazavov et al. 2009 Phys. Rev. D 80, 014504.

[16] T. Hirano and M. Gyulassy 2006 Nucl. Phys. A 76971.

[17] P. Danielewicz and M. Gyulassy 1985 Phys. Rev. D 3153.

[18] I. Bautista, J. Dias de Deus and C. Pajares arXiv:hep-ph/1102:3837.

[19] M. Prakash et al.1993 Phys. Rept. 227321.

[20] P. K. Kovtun, D. T. Son and A. O. Starinets 2005 Phys. Rev. Lett. C 94111601.

[21] P. Petreczky, Lattice 2012, Cairns, Australia, 24 - 30 June 2012. 